\title{
Commentary
}

\section{Immunization as a tool to combat antimicrobial resistance}

\author{
Spika $\mathbf{J}^{1^{\star}}$, Rud EW $^{1,2}$ \\ ${ }^{1}$ Centre for Immunization and Respiratory Infectious Diseases, Public Health Agency of Canada, Ottawa, ON \\ ${ }^{2}$ University of Ottawa, Faculty of Medicine, Department of Pathology and Laboratory Medicine, Ottawa, ON \\ *Correspondence: john.spika@phac-aspc.gc.ca
}

\begin{abstract}
Vaccines and immunization programs can play a key role in addressing the growing challenge of antimicrobial resistance (AMR). Amongst the high priority vaccines in development are several AMR pathogens, including: Clostridium difficile, Staphylococcus aureus, Streptococcus pneumoniae, Mycobacterium tuberculosis and Neisseria gonorrhoeae. There is evidence that vaccination can reduce the prevalence of AMR microbes, as demonstrated by both pneumococcal and Haemophilus influenza b vaccines. Research continues on many vaccine-preventable diseases, many of these AMR pathogens, including HIV and universal influenza vaccines. Not only do vaccines prevent infections, they can also prevent secondary opportunistic infections from AMR microbes-for example, bacterial pneumonia following influenza infections. The reduced need to treat these opportunistic infections would also mitigate the advance of AMR microbes in our communities. However, vaccines are not a panacea. One downside to the use of vaccines to address AMR is vaccine hesitancy, which undermines efforts to achieve herd immunity, but this is being increasingly addressed by public health education campaigns.
\end{abstract}

\section{Introduction}

Is the 21st century going to be remembered as the beginning of the post-antibiotic era? With each passing year, our list of effective antimicrobials is slowly shrinking, as more microbes become resistant in both the human and animal settings. At the same time, fewer antimicrobials are being brought to the market. Microbes by their nature continually adapt to survive the antimicrobial treatments we use to combat them, resulting in an ever increasing level of antimicrobial resistance (AMR).

The rate of methicillin-resistant Staphylococcus aureus (MRSA) infections among hospitalized patients in Canada increased nine-fold from 1995 to 2009 (0.4 - 3.8 per 10,000 patient days) (1-3). With concerted efforts, rates have been slowly decreasing but in 2014 they were still reported at 2.8 per 10,000 patient days (2). In 2012, 33\% of MRSA infections identified in hospitalized patients were acquired in the community, compared to $17 \%$ in 1995 (3). In northern Canadian communities, community-associated MRSA infection rates have been higher than anywhere else in North America (4).

Each year in Canada, more than 18,000 hospitalized patients acquire infections that are resistant to antimicrobials. The incidence rates, though relatively low for vancomycin-resistant Enterococci (VRE) infections in Canada, have slightly increased from 2009 to 2013 (0.31 and 0.52 per 10,000 patient days, respectively) (2). Deaths directly and indirectly related to Clostridium difficile infection (attributable mortality) alone increased three-fold from 1.5\% to 5.7\% between 1997 and 2005, respectively (5-7); recent efforts have kept this relatively stable from 2011-2014 (2).

The prevalence of microbes with resistance to more than one antimicrobial compound and our general lack of new antimicrobial compounds have heightened the concern on how to manage AMR in the future. As an immediate response, we urgently have to look for new antimicrobials, but also consider other control measures, like the use of vaccination to develop herd immunity against the microbes that have developed AMR. 
The purpose of this article is to highlight the role that vaccines and immunization programs could play in the battle against AMR pathogens.

\section{The case for vaccines}

Vaccines are undoubtedly one of the best investments in health. Immunization programs have contributed enormously to reducing the burden of infectious diseases, and are responsible for much of the falling rates of morbidity and mortality worldwide. In December 2010, global health leaders committed to making the next 10 years the Decade of Vaccines-to ensure discovery, development and delivery of lifesaving vaccines globally, especially to the poorest countries. Innovative vaccine approaches are under development and being prioritized to include some of the most significant threats posed by AMR, through the development of a Canadian Action Plan on Vaccine Research, Innovation and Development, the Global Vaccine Action Plan (8), and many other international efforts (9) to reduce the impact of vaccine-preventable diseases. Amongst the high priority vaccines in the research and development pipelines are several AMR pathogens, including: Clostridium difficile, Staphylococcus aureus, Streptococcus pneumoniae, Mycobacterium tuberculosis, Neisseria gonorrhoeae, HIV, and influenza.

\section{Examples of success}

There is evidence that vaccination can reduce the prevalence of AMR microbes. In South Africa, a 7-valent pneumococcal conjugate vaccine (PCV7) was introduced in 2009 and replaced with a 13-valent vaccine (PCV13) in 2011. In 2012, it was estimated that 81\% of 12-month-old children had received three doses of the vaccine. Prior to vaccination, $83 \%$ of multidrug-resistant pneumococcal disease isolates were serotypes contained in PCV7. From 2009 to 2012, amongst children younger than 2 years of age, the rate of invasive pneumococcal disease caused by penicillin-resistant isolates declined by $82 \%$, ceftriaxone-resistant isolates by $85 \%$, and multidrug-resistant variants by $84 \%(10)$. The pneumococcal vaccine programs in children have had an additional impact on the capsular types infecting adults, thus a broader range of impact than just the targeted age group.

Before the Hib vaccine was developed, up to $30 \%$ of Haemophilus influenzae type b (Hib) bacteria, which used to be a common cause of meningitis in young children, had become resistant to amoxicillin, a commonly used antibiotic. Since the introduction of Hib vaccines, the number of Haemophilus influenza type $b$ infections caused by drug-sensitive and drug-resistant bacteria has decreased by more than $96 \%$ in infants and children.

Vaccines not only help to prevent a targeted infection, they may prevent associated opportunistic infections as well. The effect of immunization on the morbidity and mortality associated with opportunistic infectionsmany of these AMR microbes-has a considerable impact on public health, for example, the prevention of bacterial pneumonia following influenza infections. The reduced need to treat for these opportunistic infections also has a direct impact on the further development of multidrug resistant AMR microbes and increased incidence of AMR microbes in our communities.

\section{New and better vaccines}

A safe and effective prophylactic or therapeutic HIV vaccine could dramatically change the lives of those susceptible to this virus and alleviate the economic burden associated with HIV infections worldwide. Microbes by their nature are very adaptable to survival challenges, whether that be avoiding the host's immunological responses or evolving rapid resistance to antimicrobials. The history of HIV and the development of drug resistance to successive iterations of new drugs are legendary. However, the use of drug cocktails and tailoring treatment regimens to individuals have helped increase the lifespan of those infected from the bleak days of the 1980 s to today. That being said, daily drug treatment is a demanding regime and not without adverse drug effects. Non-adherence to this regime can lead to the development of drug resistance. The pursuit of a safe and effective HIV vaccine has now been explored by many excellent research groups for close to 30 years, but as yet no highly effective vaccine exists. Hence, for some infectious diseases, vaccines may not be the most effective strategy in the short term and should not be seen as the sole response to AMR development. 
Until a universal influenza vaccine is developed, every year we have to prepare for a new influenza season, with a new combination of influenza A and B vaccines. Not only is genetic drift and shift an ever-looming problem, but resistance to the antivirals used to treat influenza infections can develop. Fortunately, most influenza $A$ and $B$ virus strains are susceptible to the neuraminidase inhibitor antiviral medications, oseltamivir, zanamivir and peramivir $(11,12)$. However, sporadic oseltamivir-resistant 2009 H1N1 virus infections have been identified, including with rare episodes of limited transmission (13-17), but the public health impact has been limited to date. During the 2013-2014 season, $98.2 \%$ of the 2009 H1N1 viruses tested for surveillance were susceptible to oseltamivir, and $100 \%$ of the $2009 \mathrm{H} 1 \mathrm{~N} 1$ viruses tested were susceptible to zanamivir. Additional sporadic cases of oseltamivir-resistant $2009 \mathrm{H} 1 \mathrm{~N} 1$ virus infection can be expected, and ongoing surveillance for oseltamivir resistance among influenza viruses is essential for public health since oseltamivir is the most widely used antiviral medication. Though combination therapy approaches can be used, new antivirals and other approaches to combating influenza are needed. The seasonal and pandemic vaccine programs can prevent influenza virus infections, dramatically reducing the likelihood of developing antiviral resistance. However, the current vaccines have variable vaccine efficacy, as we are witnessing in the 2014-2015 influenza season in Canada, requiring much more research and development to enhance their effectiveness, especially in the very young and the elderly. The use of higher vaccine doses and the addition of new adjuvants to existing vaccines may increase vaccine efficacy, but the "Holy Grail" would be the development of universal influenza vaccines which would protect against influenza in the face of virus drift and possibly even virus shifts that occur over time. These are being explored by several companies and research groups at this time, but this virus remains a global public health challenge.

\section{Challenges}

Ironically, in the very countries that have benefited the most from national immunization programs, there has been a loss of public confidence in vaccines by a small minority of people. Personal decision making related to vaccine acceptance is not driven by scientific nor economic arguments, but by a mix of psychological, sociocultural and political factors, all of which need to be understood and taken into account by policy and other decision makers. Public trust in vaccines is very complex and building trust depends on understanding vaccine perception and vaccine risks, historical experiences, religious or political affiliations, and socioeconomic status. Although providing accurate, scientifically based evidence on the risk-benefit ratios of vaccines is important, it is not enough to address the growing gap between current levels of public confidence in vaccines and levels of trust needed to ensure adequate and sustained vaccine coverage and long-lasting herd immunity. The recent measles outbreaks in North America and Europe in many whom have not been vaccinated by choice has highlighted the vaccine hesitancy issue. Further research into all aspects of vaccine hesitancy will help inform decision making on immunization and its importance in the fight against infectious diseases, including AMR microbes.

\section{Conclusion}

Vaccines have been one of the best public health tools in our fight against the impact of infectious diseases worldwide. Vaccines have played a paramount role in the eradication of smallpox and in bringing polio and measles close to elimination. Could vaccines show a similar global public health impact on AMR microbes? Government, industry, academia and non-governmental organizations worldwide are engaging more and more in the battle against AMR microbes. Though the pursuit of new effective antibiotics is crucial in this fight, other treatment and preventive approaches are needed to prevent the spread of AMR microbes. Development of new and improved vaccines may be crucial in our fight against AMR microbes and for maintaining and enhancing public health worldwide. However, vaccines can only be effective if the populations they are designed to protect agree to be vaccinated in the numbers required to induce herd immunity. Addressing the issue of vaccine hesitancy, through active education campaigns and other approaches, is crucial in the ongoing fight against all vaccine-preventable diseases including those that have developed resistance to our antimicrobial armada. 


\section{Acknowledgements}

The authors would like to acknowledge the contribution of Gina Charos, Director, Immunization Programs and Promotion Division, Centre for Immunization and Respiratory Infectious Diseases, Public Health Agency of Canada, for reviewing and providing comments on this article.

\section{Conflict of interest}

None

\section{References}

(1) Simor AE, Gilbert N, Gravel D, Mulvey MR, Bryce E, Loeb M, et al. Methicillin-Resistant Staphylococcus aureus in Canada: National surveillance and changing epidemiology, 1995-2007. Infect Control Hosp Epidemiol. 2010 Apr;31(4):348-56.

(2) Public Health Agency of Canada. Antimicrobial Resistant Organisms (ARO) Surveillance Report-2009-2014: Surveillance Report for Data from January 1, 2009 to December 31, 2014. Table 2.2. Updated July 2015. http://dev.healthycanadians.gc.ca/publications/drugs-products-medicaments-produits/antimicrobial-summarysommaire-antimicrobien/index-eng.php

(3) Public Health Agency of Canada. Methicillin-resistant Staphylococcus aureus in Canadian acute-care hospitals: Surveillance Report January 1, 2008 to December 31, 2012. Centre for Communicable Diseases and Infection Control, Public Health Agency of Canada, 2014. http://www.phac-aspc.gc.ca/nois-sinp/projects/aro-mra-execeng.php

(4) Golding GR, Quinn B, Bergstrom K, Stockdale D, Woods S, Nsungu M, et al. Community-based educational intervention to limit the dissemination of community-associated methicillin-resistant Staphylococcus aureus in Northern Saskatchewan, Canada. BMC Public Health. 2012;12(1):15.

(5) Gravel D, Miller M, Simor A, Taylor G, Gardam M, McGeer A, et al; Canadian Nosocomial Infection Surveillance Program. Health care-associated Clostridium difficile infection in adults admitted to acute care hospitals in Canada: A Canadian Nosocomial Infection Surveillance Program study. Clin Infect Dis. 2009;48(5):568-76.

(6) Public Health Agency of Canada. Healthcare-associated Clostridium Difficile Infections in Canadian Acute-care Hospitals: Surveillance Report January 1, 2007 to December 31, 2012. Centre for Communicable Diseases and Infection Control, Public Health Agency of Canada, 2014. http://www.phac-aspc.gc.ca/id-mi/c-difficile-sum-reseng.php

(7) Miller MA, Hyland M, Ofner-Agostini M, Gourdeau M, Ishak M. Morbidity, mortality, and healthcare burden of nosocomial Clostridium difficile_associated diarrhea in Canadian hospitals. Infect Control Hosp Epidemiol 2002;23:137-40

(8) World Health Organization (WHO). Global Vaccine Action Plan 2011-2020. Geneva: WHO; 2013. http://www.who.int/immunization/global_vaccine_action_plan/en/

(9) Gavi_Global Vaccine Alliance. Gavi's mission. http://www.gavi.org/about/mission/

(10) von Gottberg A, de Gouveia L, Tempia S, Quan V, Meiring S, von Mollendorf C et al. Effects of vaccination on invasive pneumococcal disease in South Africa. N Engl J Med. 2014;371(20):1889-99.

(11) Centers for Disease Control and Prevention (CDC). FluView-Weekly U.S. Influenza Surveillance Report. 2014-2015 Influenza Season Week 5 ending February 7, 2015. Atlanta, GA: U.S. Department of Health and Human Services, CDC; 2015. http://www.cdc.gov/flu/weekly

(12) Public Health Agency of Canada (PHAC). FluWatch report: February 1 to February 7, 2015 (Week 5). Ottawa: PHAC; updated 2015 Feb 13. http://www.phac-aspc.gc.ca/fluwatch/14-15/w05_15/index-eng.php\#a_4

(13) Baz M, Abed Y, Papenburg J, Bouhy X, Hamelin ME, Boivin G. Emergence of oseltamivir-resistant pandemic H1N1 virus during prophylaxis. New Engl J Med. 2009;361:2296-7.

(14) Le QM, Wertheim HF, Tran ND, van Doorn HR, Nguyen TH, Horby P. A community cluster of oseltamivirresistant cases of 2009 H1N1 influenza. New Engl J Med. 2010;362:86-7.

(15) Centers for Disease Control and Prevention. Update: Influenza activity-United States, 2009-10 season. Morbidity and Mortality Weekly Report (MMWR). 2010 Jul 30;59(29):901-8. http://www.cdc.gov/mmwr/preview/mmwrhtml/mm5929a2.htm

(16) Centers for Disease Control and Prevention. Oseltamivir-resistant novel influenza A (H1N1) virus infection in two immunosuppressed patients-Seattle, Washington, 2009. Morbidity and Mortality Weekly Report (MMWR). 2009 Aug 21;58(32):893-6. http://www.cdc.gov/mmwr/preview/mmwrhtml/mm5832a3.htm

(17) Centers for Disease Control and Prevention. Oseltamivir-resistant 2009 pandemic influenza A (H1N1) virus infection in two summer campers receiving prophylaxis-North Carolina, 2009. Morbidity and Mortality Weekly Report (MMWR). 2009 Sep 11;58(35):969-72. http://www.cdc.gov/mmwr/preview/mmwrhtml/mm5835a1.htm 